\title{
Influence of Test Procedures on the Thermomechanical Properties of a 55NiTi Shape Memory Alloy
}

\author{
Santo A. Padula, II ${ }^{\mathrm{a}}$, Darrell J. Gaydosh ${ }^{\mathrm{b}}$, Ronald D. Noebe ${ }^{\mathrm{a}}$, Glen S. Bigelow ${ }^{\mathrm{a}}$, Anita Garg ${ }^{\mathrm{c}}$, \\ Dimitris Lagoudas ${ }^{\mathrm{d}}$, Ibrahim Karaman ${ }^{\mathrm{d}}$, Kadri C. Atli ${ }^{\mathrm{d}}$ \\ ${ }^{a}$ N.A.S.A. Glenn Research Center, 21000 Brookpark Rd., Cleveland, OH, USA 44135; \\ bohio Aerospace Institute, 22800 Cedar Point Rd., Cleveland, OH, USA 44142; \\ ${ }^{\circ}$ University of Toledo, 2801 West Bancroft Ave., Toledo, OH, USA 43606; \\ ${ }^{\mathrm{d}}$ Texas A\&M University, College Station, TX, USA 77843
}

\begin{abstract}
Over the past few decades, binary NiTi shape memory alloys have received attention due to their unique mechanical characteristics, leading to their potential use in low-temperature, solid-state actuator applications. However, prior to using these materials for such applications, the physical response of these systems to mechanical and thermal stimuli must be thoroughly understood and modeled to aid designers in developing SMA-enabled systems. Even though shape memory alloys have been around for almost five decades, very little effort has been made to standardize testing procedures. Although some standards for measuring the transformation temperatures of SMA's are available, no real standards exist for determining the various mechanical and thermomechanical properties that govern the usefulness of these unique materials. Consequently, this study involved testing a 55NiTi alloy using a variety of different test methodologies. All samples tested were taken from the same heat and batch to remove the influence of sample pedigree on the observed results. When the material was tested under constant-stress, thermal-cycle conditions, variations in the characteristic material responses were observed, depending on test methodology. The transformation strain and irreversible strain were impacted more than the transformation temperatures, which only showed an affect with regard to applied external stress. In some cases, test methodology altered the transformation strain by $0.005-0.01 \mathrm{~mm} / \mathrm{mm}$, which translates into a difference in work output capability of approximately $2 \mathrm{~J} / \mathrm{cm}^{3}\left(290 \mathrm{in} \cdot 1 \mathrm{bf} / \mathrm{in}^{3}\right)$. These results indicate the need for the development of testing standards so that meaningful data can be generated and successfully incorporated into viable models and hardware. The use of consistent testing procedures is also important when comparing results from one research organization to another. To this end, differences in the observed responses will be presented, contrasted and rationalized, in hopes of eventually developing standardized testing procedures for shape memory alloys.
\end{abstract}

Keywords: shape memory alloy, NiTi, thermomechanical behavior, actuator, testing standards, transformation temperature, transformation strain, work output.

\section{INTRODUCTION}

In recent years, the interest in shape memory alloys for high-force actuation has grown due to the increased viability and potential weight and space savings of these solid-state actuators compared to their traditional motor, hydraulic, pneumatic or solenoid counterparts. Although there is substantial interest in shape memory alloys, information pertinent to design is scarce. To gain the information that is needed to address this shortfall, more constitutive testing is required. However, traditional testing standards are not necessarily adequate for this unique class of materials and new standards may, therefore, need to be developed. Although some testing standards do exist for shape memory alloys[1, 2, 3], these standards were predominately developed for superelastic materials and in the majority of cases have limited applicability [2,3], are insufficient for bulk actuator materials due to the sample sizes tested[1] or are only qualitative in nature[2]. Additionally, no testing standards exist for the determination of shape memory behavior under stress free or constant stress conditions, making it almost impossible to compare results from one material to the next, let alone from one research group to the next. Until this changes, it will be almost impossible to transition information gained from constitutive testing into accurate design data that can be used for component development. 
To make this transition and begin developing testing standards, more must be known about the factors that can affect shape memory behavior. However, the factors that are important to traditional monolithic deformation behavior may or may not be the same as those that influence shape memory response. In keeping with this notion, it is important to critically assess all factors that may influence the observed deformation response of SMA materials. Only then can testing standards that provide true constitutive behavior be developed. To this end, a number of testing methodologies were applied to the same batch of material to illustrate the influence of test methodology on the observed response of a baseline 55NiTi alloy. The results clearly show that testing standards need to be developed for characterizing SMA actuator materials or design with these materials will remain predominately empirical.

\section{PROCEDURES}

\subsection{Material}

The material used in this study was a commercially available, binary NiTi alloy produced by Special Metals, New Hartford, New York, with a fully annealed ingot $\mathrm{A}_{\mathrm{s}}$ temperature of $95+/-5{ }^{\circ} \mathrm{C}$, henceforth designated as $55 \mathrm{NiTi}$. In this case 55 refers to the $\mathrm{wt} \% \mathrm{Ni}$ in the alloy. In terms of at $\%$, the stoichiometry of the alloy is $\mathrm{Ni}_{49.9} \mathrm{Ti}_{50.1}$, which is optimized for high transformation temperature. The material was delivered as $10 \mathrm{~mm}$ diameter rods of varying lengths in the hotrolled/hot-drawn and hot-straightened condition. No further information on the specifics of the thermomechanical processing used to fabricate the rod was available, as specific processing details are deemed proprietary by the vendor.

\subsection{Constant Stress, Thermal Cycle Testing}

Subsequent to load-biased, thermal-cycle testing, two no-load or stress-free thermal cycles were performed on the "asmachined" specimen prior to performing any other operation. The intent of these no-load thermal cycles was to relieve any internal stresses resulting from the machining operation. Once the no-load cycles were complete, the specimen was tested using one of the protocols described below.

\section{Constant Stress, Thermal Cycling Performed in Series (Series Load-bias):}

A "series" load-biased thermal cycle test consisted of loading the stress-relieved specimen to a predetermined stress at a strain rate of $1 \times 10^{-4} \mathrm{~s}^{-1}$ using strain control. Once the desired stress level was achieved, the controller was switched into load control and the stress was held constant. The specimen was then thermally cycled from room temperature (RT) to the desired upper temperature and back for two complete thermal cycles. After completing the two thermal cycles, the controller was switched back to strain control and the specimen was strained until the next higher stress level was achieved. Once the desired stress level was achieved, the control-mode was switched back to load control and the thermal cycling process was repeated. This process of loading in strain control followed by thermal cycling under constant stress was repeated until all desired stress levels were completed using the same specimen. Stress levels from 50-300 MPa (in $50 \mathrm{MPa}$ increments) were assessed. To determine the effect of upper cycle temperature on the observed load-biased strain-temperature response, upper cycle temperatures of 165 and $200{ }^{\circ} \mathrm{C}$ were used. In all cases, only the second heating and cooling cycle for a given stress was used for analysis. This second heating and cooling cycle was chosen because of the nature of the processes involved in the response. When a stress is applied to the material or an increment in the applied stress is made, significant changes in the martensite variant structure can result during the first thermal cycle under stress. Hence, the first cycle doesn't exhibit the same characteristics as subsequent cycles at a given stress and is therefore not used for analysis. Thus, the second cycle was used for analysis as it not only exhibits the properties of the reoriented structure, but also is the minimum number of cycles that can be run in a constant-stress, thermal-cycle test to ascertain behavior. 


\section{Constant Stress, Thermal Cycling Performed on Individual Samples (Single Load-bias):}

The "single" load-biased thermal cycling experiments were similar to the "series" load bias experiments, however, in the experiments denoted as "single", each stress level was run on an entirely different stress-relieved specimen with no prior testing history. This is in contrast to the experiments denoted "series" where all stress levels were run on the same specimen. Testing for "single" load-bias experimentation was performed at 100, 200 and $300 \mathrm{MPa}$. An upper cycle temperature of $200{ }^{\circ} \mathrm{C}$ was used for the thermal cycle and the results were compared against those obtained for experiments conducted in "series". Again, only the second heating and cooling cycle under stress was used for the purpose of analysis.

\section{Martensite vs. Austenite Initial Loading:}

The "single" load-biased thermal-cycling methodology was also used to assess the effect of the temperature at which stress was first applied on the subsequent strain-temperature response of the material. In the previously defined "single" load-biased thermal cycling tests, the load was initially applied at room temperature while the material was in the martensitic condition. Alternative tests were performed whereby the sample was first heated, under stress-free conditions, to the upper temperature of $200{ }^{\circ} \mathrm{C}$ prior to applying the load, so that the load was first applied while the material was in the austenitic state. The remainder of the testing was performed in an identical manner to the original "single" load-biased thermal-cycling experiment, at constant stress levels of 100, 200 and $300 \mathrm{MPa}$. In both cases, martensite loaded vs. austenite loaded, only the data resulting from the second heating and cooling cycle was used for analysis.

\section{RESULTS \& DISCUSSION}

Although basic mechanical and thermal properties are important, the load-biased strain-temperature response is what governs the materials ability to do work and it is this constitutive behavior that must be understood to utilize SMA's for actuator applications. Although many load-biased, thermal-cycling experiments have been performed on a number of different material systems in the past, no standardized methodology has been developed. Thus, results from this type of test using a number of different methodologies on the same material were compared in an attempt to understand the impact of different test procedures.

A number of different material properties are generated from a constant-stress thermal cycle test. Figure 1 shows the typical parameters that are assessed. Transformation temperatures were determined by making linear fits through three portions of the heating and cooling curves; namely, the martensite, martensite+austenite and austenite portions.

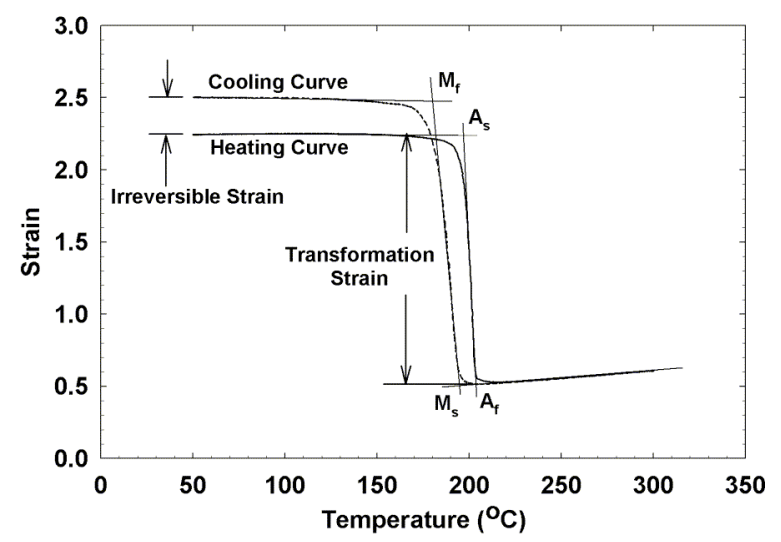

Figure 1. Representation of how each of the parameters are analyzed using strain-temperature data generated during a constant-stress, thermal-cycling experiment. 
These linear fits were then extrapolated to determine the intersection points, which were taken to be the onset and finish of the transformation. Likewise, the transformation strain, which governs the materials ability to do work, was determined by taking the difference in axial strain measured by the intersection points for the heating portion of the thermal cycle. This transformation strain was then used to calculate normalized work by multiplying it by the stress maintained during thermal cycling. Finally, the amount of irreversible strain was determined by taking the difference in axial strain observed between the beginning and end of the thermal cycle at room temperature. This parameter is used as a measure of the dimensional stability of the material and ideally would be zero.

\subsection{Effect of Upper Cycle Temperature}

The upper most temperature of the thermal cycle, referred to here as the upper cycle temperature, may be the simplest factor that is most often overlooked in terms of impact on subsequent test results. No matter which alloy one is testing, the choice of where to end the thermal cycle is currently arbitrary but is usually chosen as some finite amount beyond the austenite finish $\left(\mathrm{A}_{\mathrm{f}}\right)$ temperature, where the transformation to the austenite phase is presumed complete. As can be seen in Figures $2 \mathrm{a}$ and $2 \mathrm{~b}$, the arbitrary choosing of $165{ }^{\circ} \mathrm{C}\left(\mathrm{A}_{\mathrm{f}}+50\right)$ and $200{ }^{\circ} \mathrm{C}\left(\mathrm{A}_{\mathrm{f}}+85\right)$, where $\mathrm{A}_{\mathrm{f}}$ was determined to be $\sim 115$ ${ }^{\circ} \mathrm{C}$ by differential scanning calorimetry, resulted in different strain-temperature responses. In this case, tests were conducted in "series", where each successive bias-stress level was performed on the same specimen. By the end of the test, a $0.01 \mathrm{~mm} / \mathrm{mm}$ difference in accumulated strain was observed between the two samples. This difference in ending strain levels is potentially important both from a modeling perspective as well as from a design perspective. However, the real impact of the upper cycle temperature is more clearly evident when one looks at the specific design parameters extracted from the load-biased thermal-cycling data.

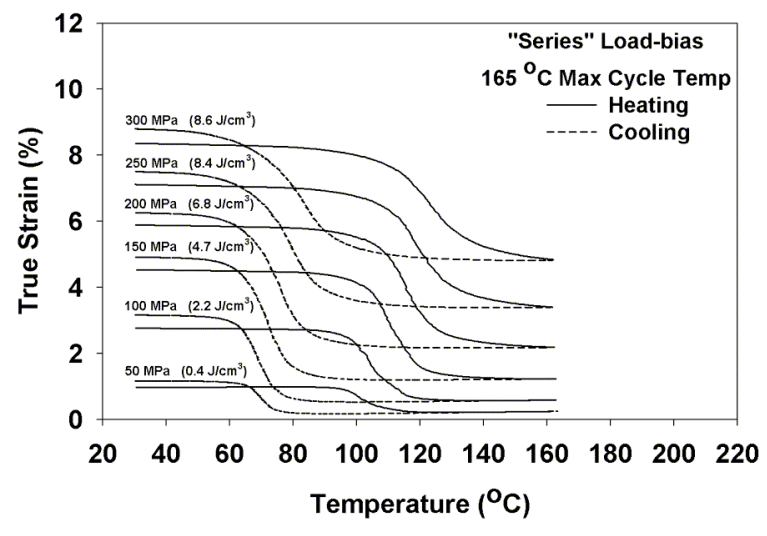

(a)

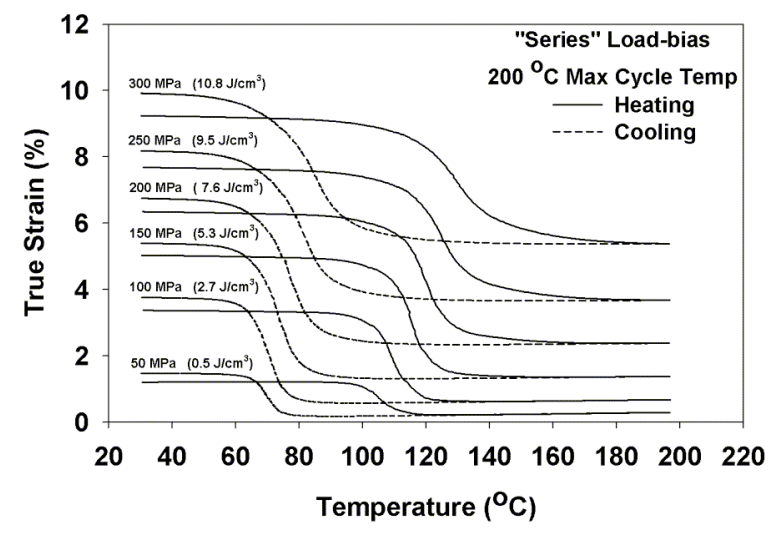

(b)

Figure 2. The load-biased, strain-temperature response of 55NiTi for an upper cycle temperature of (a) $165^{\circ} \mathrm{C}$ and (b) $200{ }^{\circ} \mathrm{C}$.

The effect of the upper cycle temperature on the transformation strain, irreversible strain, and transformation temperature as a function of applied stress is shown in Figures $3 \mathrm{a}, 3 \mathrm{~b}$, and $3 \mathrm{c}$, respectively. The transformation strain, which is directly related to the work output of the material, increased to a maximum at around $200 \mathrm{MPa}$ and subsequently decreased with further increases in the applied stress, regardless of the upper temperature used for the thermal cycling. This peak in transformation strain with stress is common to NiTi alloys [4,5] and is due to competing factors. As the stress level increases, more martensite is favorably aligned during the cooling portion of the cycle so that the available transformation strain on heating increases, but eventually, the effect saturates as all the martensite becomes favorably oriented. When this occurs, the stress becomes large enough to begin to suppress the reverse transformation, and at these higher stress levels, slip rather than additional twinning is activated, further inhibiting the shape recovery and decreasing the transformation strain. These competing mechanisms lead to a peak in transformation strain with increasing stress level, as shown in Figure 3a. 
However, there was a significant difference in the amount of transformation strain measured at each stress level depending on upper cycle temperature, with the higher cycle temperature resulting in a greater transformation strain. This difference between the $165{ }^{\circ} \mathrm{C}$ and $200{ }^{\circ} \mathrm{C}$ conditions is believed to be due to differences in retained martensite. Retained martensite is martensite that does not convert to austenite at a given temperature above the apparent $\mathrm{A}_{\mathrm{f}}$ temperature due to stress in the material, which is a combination of both externally applied and internal residual stress. Even when the transformation appears to be complete, as is often assumed when the strain-temperature response flattens out at the elevated temperature side of the thermal cycle, retained martensite can still be present, depending on the stress and temperature conditions $[6,7]$. However, this retained martensite will eventually convert to austenite at some higher temperature. Although for both conditions the apparent $A_{f}$ temperature is essentially the same and appears to be less than $140{ }^{\circ} \mathrm{C}$ at the maximum stress tested, the additional thermal energy gained by heating to $200{ }^{\circ} \mathrm{C}$, is apparently causing additional martensite to revert to austenite, when compared to the $165^{\circ} \mathrm{C}$ condition. Hence, more austenite is available to reconvert to martensite during the subsequent cooling under load. This martensite which develops is more favorably oriented to accommodate strain on the subsequent thermal cycle, which is the one used for analysis.

In this particular case, differences in transformation strain on the order of $0.005-0.01 \mathrm{~mm} / \mathrm{mm}$ were observed. These levels of transformation strain translate into differences in work output capability on the order of $1-2 \mathrm{~J} / \mathrm{cm}^{3}(145-290$ in $\cdot \mathrm{lbf} / \mathrm{in}^{3}$ ) at stress levels greater than $200 \mathrm{MPa}$, which can be significant from an actuator standpoint and highlights the need to run tests under similar conditions to the operating environment for the actuator.

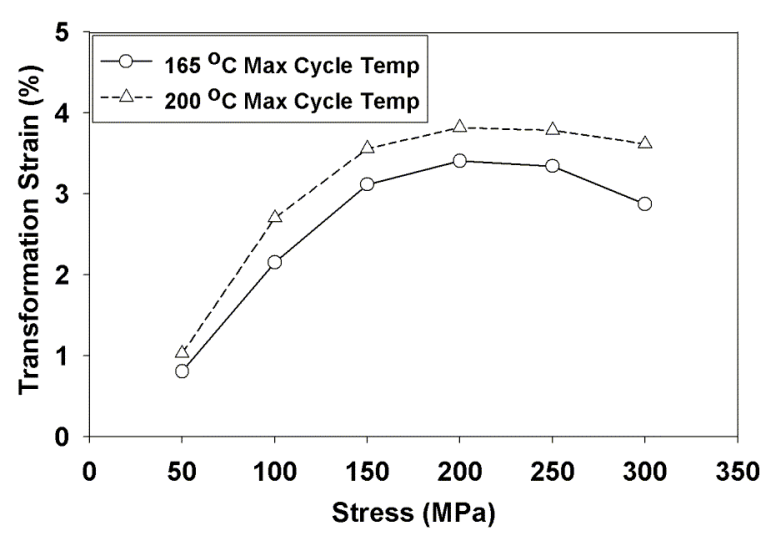

(a)

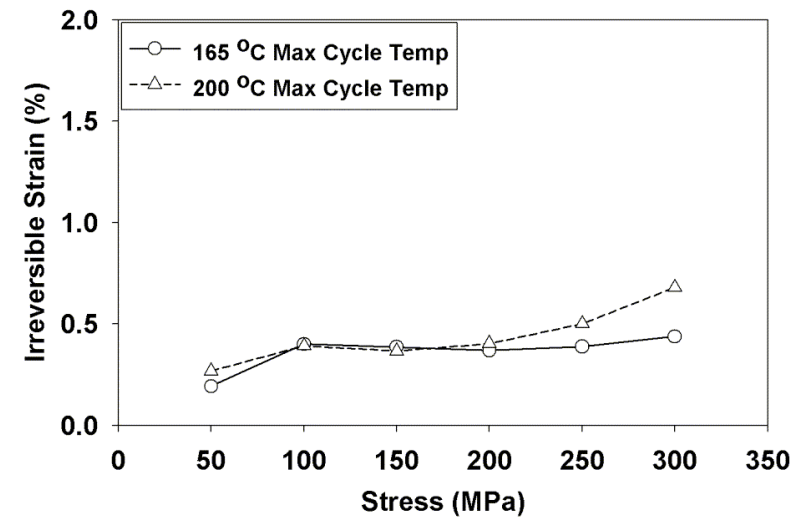

(b)

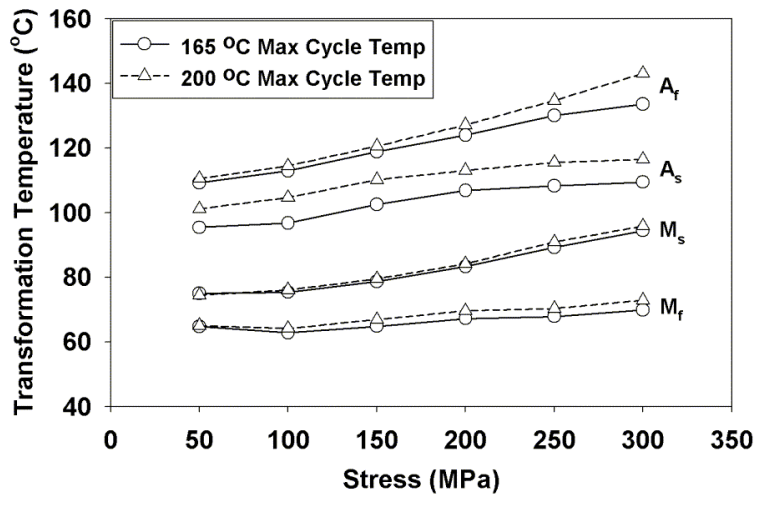

(c)

Figure 3. (a) Transformation strain, (b) irreversible strain, and (c) transformation temperatures, as a function of stress for $55 \mathrm{NiTi}$ tested to different upper-cycle temperatures. 
In contrast, there is little or no effect of upper cycle temperature on irreversible strain except at the highest stress levels encountered, as shown in Figure 3b. This irreversible strain is additional strain that is generated during the thermal cycling process that is not recovered during the reverse martensitic reaction but is presumably due to plastic or permanent deformation of the martensite and/or austenite.

The transformation temperatures for the alloy were a function of applied stress with the $A_{f}$ temperature shifting by as much as $30{ }^{\circ} \mathrm{C}$ over the stress range tested (Figure 3c). This increase in transformation temperature with stress is commonly observed and is believed to be the result of both internal elastic strain energy $[8,9,10]$ and increased frictional resistance to variant boundary movement[10] due to the applied stress. Interestingly enough, the upper temperature of the thermal cycle didn't have a dramatic effect on the transformation temperatures for the alloy, except in the case of the $\mathrm{A}_{\mathrm{s}}$ where an approximately $7{ }^{\circ} \mathrm{C}$ difference was observed at all stress levels. Although the mechanism responsible for this shift in $\mathrm{A}_{\mathrm{s}}$ with increasing upper cycle temperature is at present unclear, the repeatable nature of the shift in $\mathrm{A}_{\mathrm{s}}$ at all stress levels tested tends to support the presumption that it is a real effect. This would indicate that something associated with the increase in upper cycle temperature is making the start of the reverse transformation more difficult.

\subsection{Thermal Cycling Using "Series" vs. "Single” Loading Methodologies}

Many load-biased, shape-memory experiments are performed with successive stress levels implemented in "series" on a single sample to conserve specimens and generate as much data as possible in the shortest period of time. Unfortunately, doing so produces the added complication that history effects from the prior cycling under load could influence the subsequent response. For this reason, thermal-cycling tests were conducted on "virgin" or stress-free samples at a "single" stress level and compared to the data collected from the "series" load-biased thermal-cycling experiment (Figures $4 \mathrm{a}$ and $4 \mathrm{~b}$ ). In this case, the most notable difference in behavior due to the different testing methodologies is the amount of irreversible strain that develops from the beginning to the end of the thermal cycle, especially at the higher stress conditions as clearly shown in Figure 5 b.

This difference in the level of irreversible strain that develops during series versus single-stress thermal cycling, demonstrates the role that prior history plays in dictating the observed constitutive shape memory response. As an example, the $300 \mathrm{MPa}$ load-biased strain-temperature curve in the "series" data (Figure 4a) exhibits significantly less irreversible strain than does the comparable strain-temperature curve for the "single"-loaded case (Figure 4b). This reduction in irreversible strain at $300 \mathrm{MPa}$ for the "series"-loaded sample is the result of prior microstructural evolution that occurred when the specimen was cycled at all the lower stress levels, essentially strain hardening or strengthening the material against further irreversible deformation processes. Hence, a reduction in the amount of irreversible strain is observed when compared to the "single" cycle $300 \mathrm{MPa}$ condition, where no prior cycling and thus no microstructural evolution occurred.

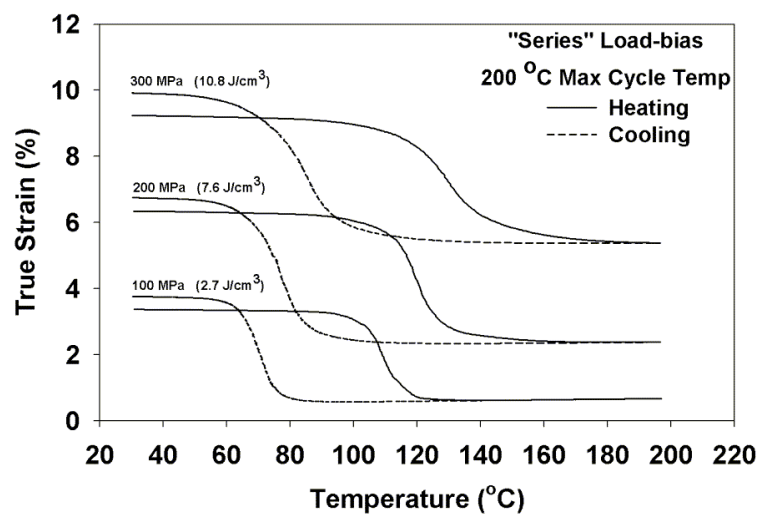

(a)

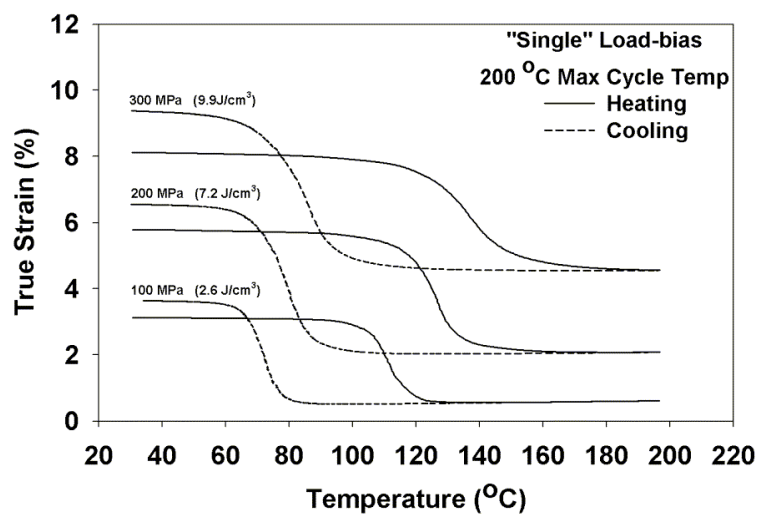

(b)

Figure 4. Comparison of load-biased, thermal-cycling results for tests run using the (a) "series" and (b) "single" cycle methodologies. Note that the additional strain-temperature cycles for the "series"-loaded condition at 50,150, and $250 \mathrm{MPa}$ are not shown to make comparisons easier but the data is included in Figure 5 for completeness. 
However, the differences in irreversible strain decrease with decreasing stress level, as would be expected, since the differences in prior history are less. For example, in the case of the $100 \mathrm{MPa}$ stress level, the differences in irreversible strain are small, as the only history difference between the two test methodologies at this point result from the two thermal cycles performed at $50 \mathrm{MPa}$ in the "series" experiment (not shown in Figure 4a). The prior thermal cycles, which dramatically reduce the irreversible strain at higher stresses in the "series"-loaded samples compared to the "single"-load tests, act to "strengthen" the microstructure and may also affect the type and number of martensite variants that are formed. While the differences are not nearly as dramatic, these same changes would also explain the slight but growing increase in transformation strain for the "series"-loaded samples compared to the "single"-load condition (Figure 5a). Similarly, these effects make it easier for the reversion reaction to occur in the "series"-loaded sample as evident from the approximately $6{ }^{\circ} \mathrm{C}$ decrease in both the $\mathrm{A}_{\mathrm{s}}$ and $\mathrm{A}_{\mathrm{f}}$ temperatures compared to the "single"-load condition at stresses greater than $100 \mathrm{MPa}$ (Figure 5c). However, no variation, greater than the expected experimental error, was observed for the $\mathrm{M}_{\mathrm{s}}$ and $\mathrm{M}_{\mathrm{f}}$ temperatures for the two test methodologies.

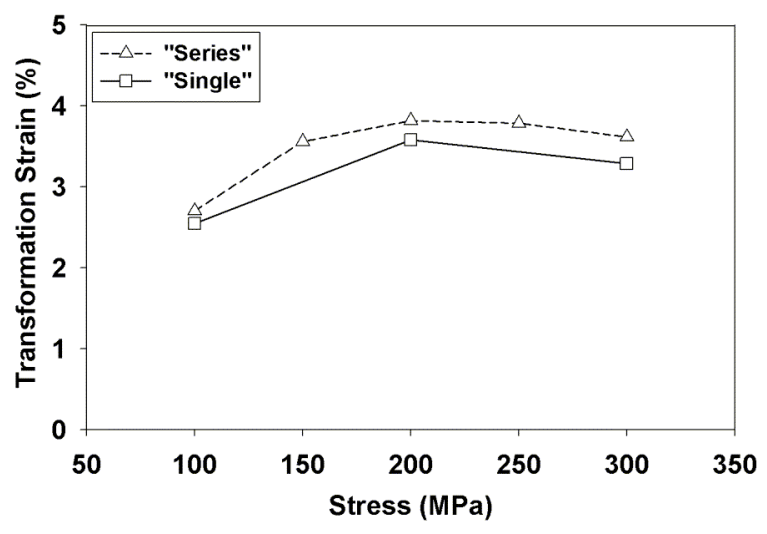

(a)

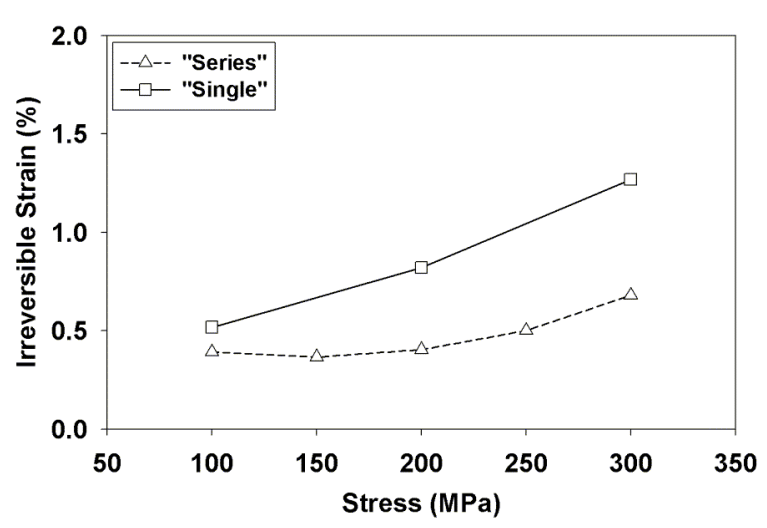

(b)

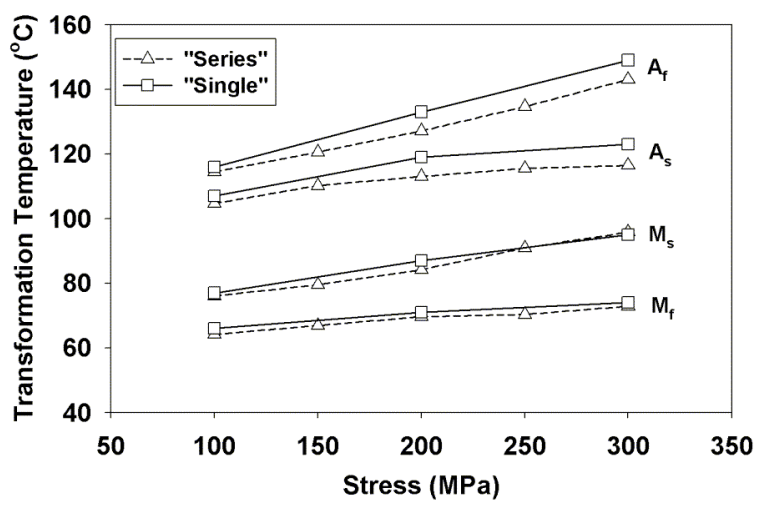

(c)

Figure 5. (a) Transformation strain, (b) irreversible strain, and (c) transformation temperatures, as influenced by loading history during testing.

In summary, although differences in the amount of irreversible strain were observed, particularly at the higher stress levels, these differences were essentially due to a strengthening effect from the previous cycles that were run in the "series" case. Also, variation of the test methodology produced minimal effect on the transformation temperatures with the effect on the measured $\mathrm{A}_{\mathrm{s}}$ and $\mathrm{A}_{\mathrm{f}}$ temperatures at higher stress being limited to a few degrees at most. Although the methodology used to perform the load-biased testing did have an observable effect on the transformation strain (and 
hence work output) of the material, the effect was minor, indicating that the "series" test is an acceptable method for screening materials and may be more realistic for cyclic, actuator-type applications.

\subsection{Martensite vs. Austenite Loading}

There is some speculation in various camps within the SMA community that differences in shape memory response may also occur if the material is initially loaded in the martensite state rather than the austenite state, prior to thermal cycling under load. Thus, the 55NiTi alloy was tested in a manner such that the behavior of specimens initially loaded in the martensite state could be directly compared to the behavior of specimens initially loaded in the austenite state. It is important to note that specimens loaded in the austenite state were out of phase with specimens loaded in the martensite state by half a thermal cycle with respect to application of the load. This half cycle shift is illustrated in Figures $6 \mathrm{a}$ and $6 \mathrm{~b}$ for clarity. Figure $6 \mathrm{a}$ represents the observed response of the material when loaded in the martensite state, the way the majority of the tests were run during this investigation. As can be seen in Figure 6a, loading occurs at room temperature (point 1) prior to the start of the initial heating cycle (point 2). The material is then heated through the transformation to the upper cycle temperature (point 3 ) and subsequently cooled under stress back to room temperature (point 4), forming a reoriented variant structure. In comparison, the material loaded in the austenite state follows the path outlined in Figure $6 \mathrm{~b}$. In this case, load control was used to maintain zero stress on the sample starting at room temperature (point 1) while heating the sample through the transformation to the upper cycle temperature (point 2). Once the upper temperature of the thermal cycle was reached, the specimen was loaded in strain control until the desired stress level was achieved (point 3). The material was then cooled through the transformation under stress back to room temperature (point 4). Hence, by point 4 in the respective tests, both specimens had cooled under stress and had formed a re-oriented variant structure. At this point, the only difference between the two specimens was the half cycle shift with respect to the temperature at which the load was first applied. Data was then taken from the second thermal cycle represented by the path from point 4 to point 5 and back to 6 on both Figures $6 \mathrm{a}$ and $6 \mathrm{~b}$, to ascertain differences in the load-biased, straintemperature responses related to loading in the martensite versus austenite states.

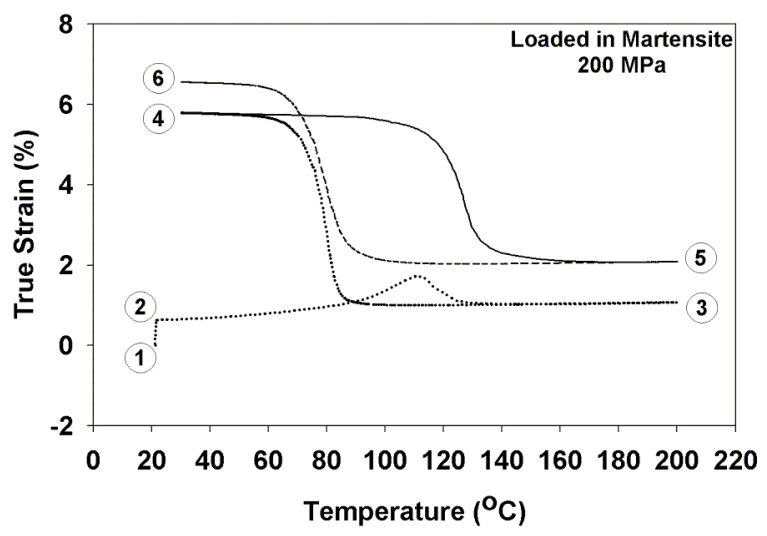

(a)

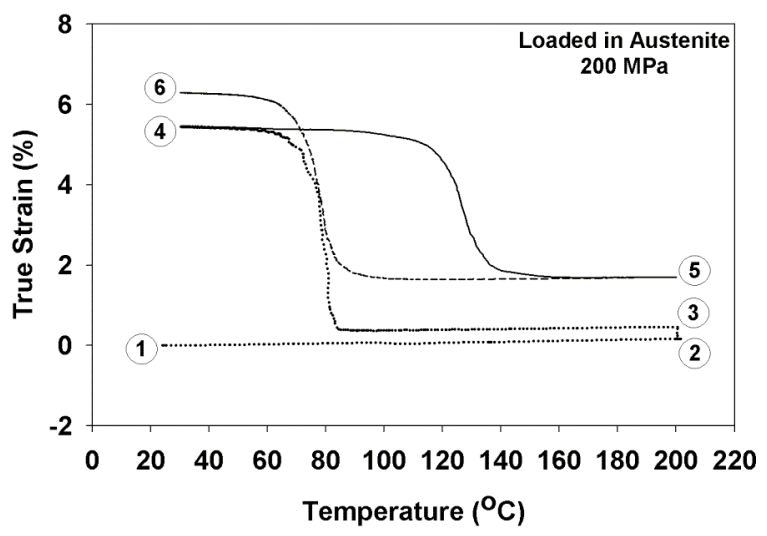

(b)

Figure 6. Examples of the thermomechanical cycles used for initially loading samples in the (a) martensite and (b) austenite states.

The entire set of load-biased thermal-cycling curves is presented in Figures $7 \mathrm{a}$ and $7 \mathrm{~b}$ and the corresponding comparisons of transformation strain, irreversible strain and transformation temperatures as a function of stress are shown in Figure 8. No significant differences in behavior were observed from loading the material in the austenite state versus the martensite state, except for the $300 \mathrm{MPa}$ biasing stress condition, which showed a greater amount of irreversible strain when loaded in the austenite state. Outside of this difference, both methodologies showed good agreement in terms of transformation strain, work output and dimensional stability (irreversible strain). 


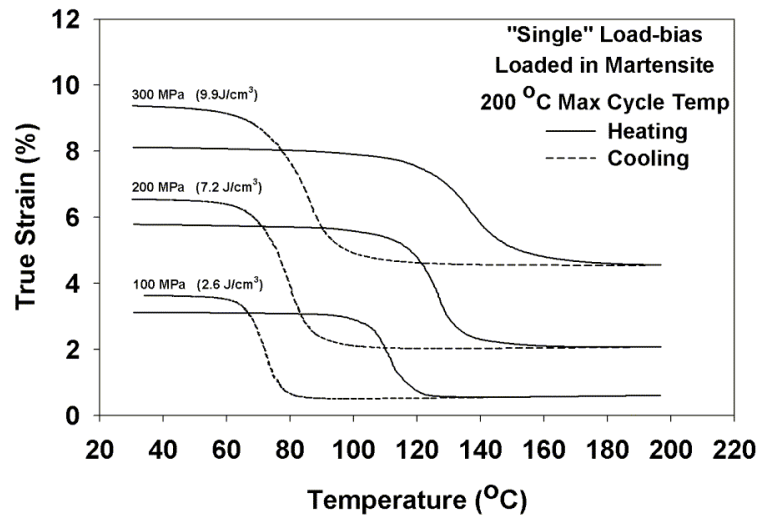

(a)

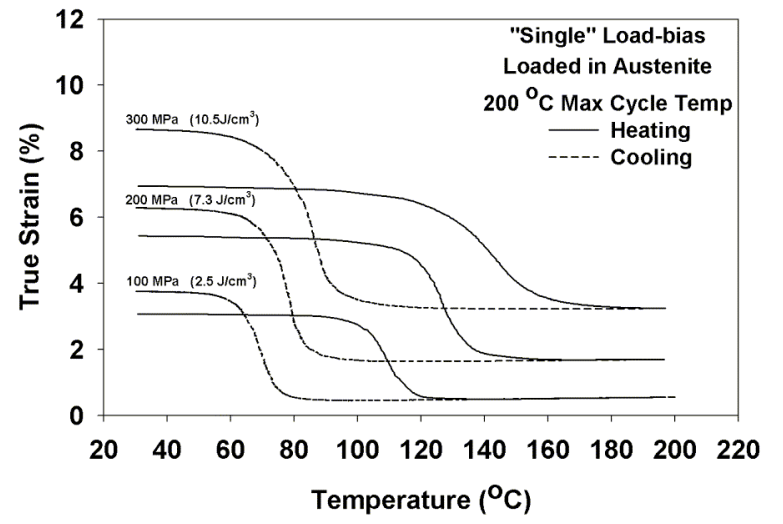

(b)

Figure 7. Comparison of "single" load-biased, thermal-cycling results when the material is first loaded in the (a) martensite and (b) austenite states.

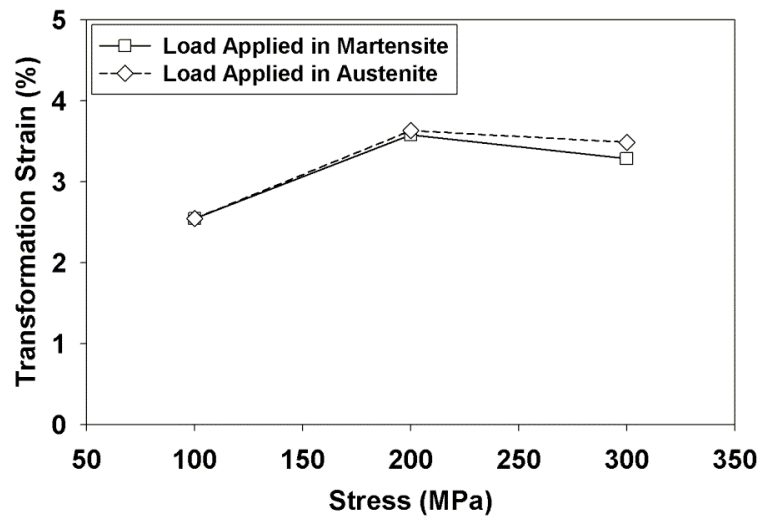

(a)

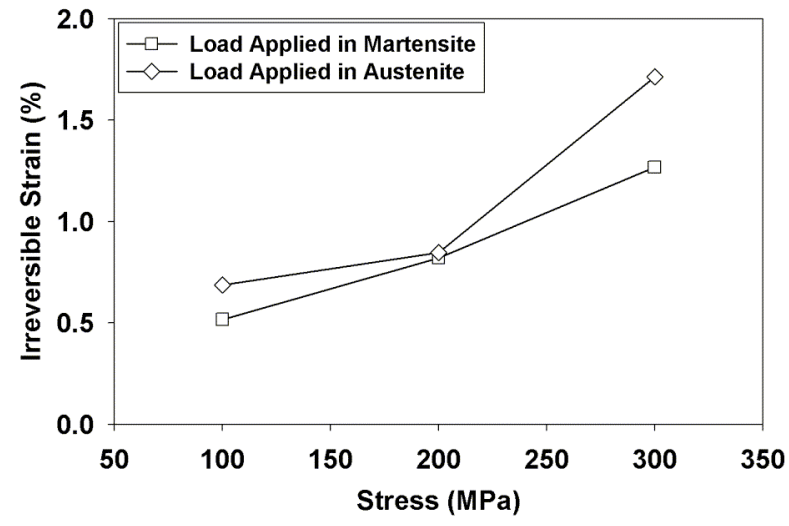

(b)

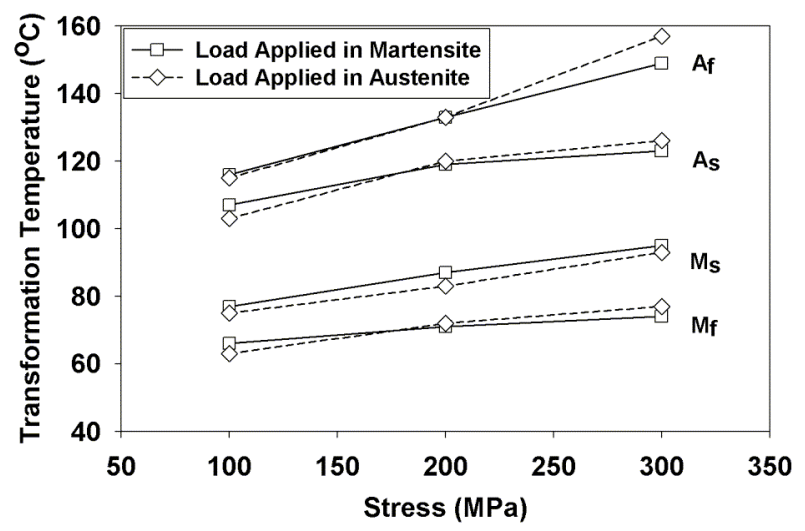

(c)

Figure 8. (a) Transformation strain, (b) irreversible strain and (c) transformation temperatures, for 55 NiTi initially loaded cold (in the martensite state) and hot (in the austenite state). 


\section{SUMMARY \& CONCLUSIONS}

The effects of various test methodologies on the load-biased strain-temperature response of a 55NiTi alloy were determined. Testing a single sample using the "series" methodology, where multiple stress levels are run on the same sample, provided fairly similar results in terms of transformation strain, work output and transformation temperature as testing a "virgin" sample at each individual stress level. The major difference observed between these two techniques was the magnitude of the irreversible strain, which was smaller in the "series"-loaded sample at stresses greater than 100 MPa due to a strengthening effect resulting from prior deformation of the sample. Outside of this difference, the "series" methodology appears to be a very useful and generally accurate screening tool for rapid evaluation of the work characteristics of shape memory alloys.

Essentially no effect, with the exception of irreversible strain at the highest stress level investigated, was observed when comparing the results on the basis of the temperature at which the material was initially loaded (whether in the martensite or austenite state). This would seem straightforward, as the only difference in the testing methodology was a half cycle difference with respect to when the load was applied, with this half cycle difference occurring prior to cooling under the applied stress. Thus, any differences would be expected to be small relative to the effects of re-orientation and plasticity that occur when the material is cooled through the transformation under stress.

Interestingly, the upper temperature of the thermal cycle had a significant effect on the response of the material, especially the transformation strain and thus work output. While not demonstrated in this study, it is believed that this effect can be attributed to the amount of retained martensite in the material and the reversion that takes place at higher cycle temperatures. Further work is needed to fully realize the implications of these affects and their underlying causes such that specific testing methodologies that ascertain the true constitutive response of the material can be developed and implemented across the SMA community. Until this is done, successful utilization of SMAs in actuator applications will remain out of reach from both a modeling and a design perspective.

\section{ACKNOWLEDGEMENTS}

The authors would like to acknowledge the stimulating discussions that have taken place with our CASMART partners, especially our colleagues at Boeing Phantom Works and Dr. Raj Vaidyanathan of the University of Central Florida. This work was supported by the N.A.S.A. Fundamental Aeronautics Program, Subsonic Fixed Wing Project.

\section{REFERENCES}

[1] ASTM F2004, "Standard Test Method for Transformation Temperature of NiTi Alloys by Thermal Analysis", ASTM Int'1, 2000 (revised 2005).

[2] ASTM F2082, "Standard Test Method for Determination of Transformation Temperature of NiTi SMA by Bend and Free Recovery”, ASTM Int'1, 2001 (revised 2006).

[3] ASTM F2516, "Standard Test Method for Tension Testing of NiTi Superelastic Materials", ASTM Int'l, 2005 (revised 2007).

[4] Duerig, T.W. et al., "Actuator and Work Production Devices", in Engineering Aspects of Shape Memory Alloys, Butterworth-Heinemann, (Boston, 1990), pp. 181-194.

[5] Cross, W.B. et al., "NITINOL Characterization Study", NASA CR-1433 (1969).

[6] Rathod, C.R., Clausen, B., Bourke, M.A.M. and Vaidyanathan, R., "Neutron diffraction investigation of hysteresis reduction and increase in linearity in the stress-strain response of superelastic NiTi", Applied Physics Letters, 88, 201919 (2006).

[7] Vaidyanathan, R. et al., (unpublished work), University of Central Florida (2007).

[8] Liu, Y. and Favier, D., "Stabilization of martensite due to shear deformation via variant reorientation in polycrystalline NiTi”, Acta Mater., 48, 3489-3499 (2000).

[9] Lin, H.C., Wu, S.K., Chou, T.S. and Kao, H.P., "The effects of cold rolling on the martensitic transformation of an equiatomic TiNi alloy", Acta Metall. Mater., 39, 2069-2080 (1991). 
[10] Lin, H.C. and Wu, S.K., "Determination of Heat of Transformation in a cold-rolled martensitic TiNi alloy", Met. Trans. A, 24A, 293-299 (1993). 